\title{
USOS Y SENTIDOS DE LAS PRÁCTICAS CORPORALES EN POLÍTICAS DE RECUPERACIÓN DEL ESPACIO PÚBLICO URBANO. LA PLATA, ARGENTINA
}

\author{
USES AND MEANINGS OF BODY PRACTICES IN POLICIES FOR URBAN PUBLIC SPACE \\ RECOVERY. LA PLATA, ARGENTINA
}

Emmanuel Ferretty ${ }^{1}$

\section{Resumen}

En este artículo se exponen algunos resultados de dos años de investigación como becario de estudio de la Comisión de Investigaciones Científicas (CIC) de la provincia de Buenos Aires, Argentina. En el plan de trabajo desarrollado se ha indagado la noción de espacio público como articulación entre políticas y prácticas corporales deportivas, recreativas y de actividad física en la ciudad de La Plata, capital de la provincia antes mencionada. El objetivo general de esta primera etapa consistió en comprender en qué consisten estas políticas de recuperación del espacio urbano, con qué sentidos se promueven prácticas corporales, qué se entiende por espacio público y cómo estos sentidos abonan cierto imaginario dominante de ciudad. La relevancia de estas políticas reside en que promueven ciertos usos corporales del espacio urbano y también constituyen las condiciones de posibilidad sobre las cuales se implementan otros planes y programas deportivos, recreativos y de actividad física orientada a la salud.

Palabras claves: usos y sentidos, prácticas corporales, políticas, espacio público, La Plata.

\section{Abstract}

This article presents few of the results of a two years research performed as a fellow student in the Comisión de Investigaciones Científicas (CIC) (Scientific Research Commission) of the Buenos Aires Province, Argentina. In the work plan developed, the notion "public space" was investigated in the relationship between policies and sport and recreational practices, and physical activity in La Plata city (capital of the mentioned province). The general goal of this first part was to understand these recovery policies of urban space, what senses body exercises are promoted with, what the term public space refers to, and how these meanings feeds some dominant imagery of the city. The importance of these policies is that they promote certain uses of urban space for body practices, and they also constitute the conditions of the possibility on which other sport and recreational plans and programs, and health-oriented physical activities are implemented.

Keywords: uses and meanings, body practices, policies, public space, La Plata.

Fecha de recepción: 10 de octubre de 2014

Fecha de aprobación: 20 de marzo de 2015

Para citar este artículo:

Ferretty, E. (2015). Usos y sentidos de las prácticas corporales en políticas de recuperación del espacio público urbano. La Plata, Argentina. Lúdica Pedagógica, 21, 43-51.

\footnotetext{
1 Profesor en Educación Física y doctorando en Comunicación (UNLP, Argentina). Filiación institucional: (1) Comisión de Investigaciones Científicas (CIC) de la provincia de Buenos Aires. (2) Instituto de Investigaciones en Humanidades y Ciencias Sociales (UNLP-Conicet). Facultad de
} Humanidades y Ciencias de la Educación. Universidad Nacional de La Plata, Argentina. Correo electrónico: eferretty@gmail.com 


\section{PUNTOS DE PARTIDA, ENFOQUE E ITINERARIOS DE INVESTIGACIÓN}

"Lo público siempre fue una zona gris [...], lo mío es de la línea municipal para adentro". José, funcionario municipal (03/12/2013)²

En principio, la hipótesis que se sostiene es que tanto los sentidos de recuperación como los sentidos que las prácticas corporales adquieren en el marco de estas políticas consisten en un intento de (re)definición material y simbólica de dónde, quién(es) y cómo es legítimo apropiarse del espacio público urbano. Este intento de fundar otro orden corporal y espacial reposa sobre un diagnóstico negativo de un pasado próximo que le sirve de justificación. En efecto, el sentido de recuperación no solo opera sobre la dimensión espacial sino también, y de modo inevitable, sobre las prácticas y los sujetos que se apropian o utilizan dichos espacios. Tal como señala Adrián Gorelik (2008), el espacio público sería la invención/ficción que articula política y forma en la ciudad.

Las sospechas que motivaron la construcción del problema de investigación son producto de la observación sistemática de ciertas transformaciones materiales en los espacios verdes de la ciudad de La Plata y, correlativamente, de cambios en los modos prácticos y corporales en que sujetos juveniles y adultos se apropiaban de estos espacios urbanos. En principio, estas observaciones se realizaron en el contexto del gobierno municipal del periodo 2007-2011 y refieren, de manera puntual, a la remodelación de plazas, parques, ramblas, paseos del casco urbano de La Plata y a la promoción de usos recreativos, deportivos y saludables de la ciudad.

Las primeras exploraciones, caracterizadas por la inmediatez de la experiencia urbana, fueron interpelaciones por las mismas obras y por las cartelerías que se instalaron temporalmente hasta su finalización. A partir de ellas, se obtuvieron algunas pistas que condujeron a otras indagaciones de índole documental e institucional, ya en el marco del plan de trabajo de beca de investigación, a partir del año 2012. En estas búsquedas se evidenció el hecho de que dichas transformaciones fueron consecuencia de la implementación a nivel

2 "José" es el nombre ficticio que se utiliza para reservar la identidad de un funcionario municipal que ocupa un cargo relevante en el marco institucional estudiado. Es decir, su importancia se fundamenta en la posición privilegiada de toma de decisiones respecto del espacio público y de la promoción de prácticas corporales. municipal y provincial de dos planes diferentes, pero homónimos, titulados "Plan de Recuperación del Espacio Público" (PREP).

Por estas razones, se comenzó interrogando a la ciudad desde una perspectiva antropológica y política: ¿Qué espacios urbanos de La Plata son "recuperados"? ¿Qué obras se realizan?; ¿Quiénes, cómo y por qué practican efectivamente dichos espacios urbanos? ¿Qué motivos justifican e impulsan estas iniciativas estatales? ¿Cuál de estos dos planes tiene mayor injerencia en la ciudad de La Plata? ¿Existen puntos de encuentro, colaboraciones, paralelismos o contradicciones políticas? ¿Qué espacios y prácticas se promueven? ¿Cómo se conciben las prácticas corporales en dichos planes?

Es decir, el interrogante se centra en los sentidos, discusiones y disputas acerca de lo público; acerca de qué y cómo es el espacio público para el Estado y, particularmente, para los actuales gobiernos de la municipalidad de La Plata y de la provincia de Buenos Aires. Ahí radica la decisión teórica de hacer un acercamiento a la noción de políticas estatales en vez de políticas públicas respecto de la necesidad, detallada por Lahera (2004), de explorar y describir las características de los gobiernos que crean e implementan dichas políticas. Así, se establece la distinción primera de una relación de fuerzas acerca de lo público que comienza pero no se agota en la esfera estatal. Asimismo, esta distinción es equivalente a plantear la problemática del espacio urbano en la del espacio público y viceversa, estableciendo los puentes tanto como las distinciones antropológicas y políticas correspondientes: la ciudad como escenario político - espacio público- y la ciudad como lugar experienciado corporalmente -espacio urbano-.

En coherencia con este propósito, el abordaje teóricometodológico se orientó hacia metodologías cualitativas de investigación (Taylor y Bogdan, 1987) y métodos no-estándar (Marradi, Archenti y Piovani, 2007) que, por sus características flexibles, permiten abordar el objeto de estudio desde referencias, perspectivas y actores diversos. Las estrategias seleccionadas permiten ir del análisis discursivo de documentos oficiales y de entrevistas con funcionarios responsables de áreas relevantes a la observación y registro de obras y de prácticas corporales realizadas en el espacio urbano. Esta dialéctica entre las instituciones/funcionarios y los protagonistas de las prácticas corporales en la 
ciudad es posible desde un enfoque etnográfico de investigación (Guber, 2001) desde el cual se apela a los estudios culturales y al campo de los estudios sociales del deporte en la "lógica de intersecciones transdisciplinarias" (Reguillo, 2012).

Como se ha mencionado, el foco de las indagaciones está encendido sobre dos procesos correlativos: por un lado, la creciente preocupación y ocupación de los gobiernos municipal y provincial en materia de infraestructura urbana para usos deportivos, recreativos y saludables de la ciudad. Por otro, la observación de usos masivos de los espacios urbanos mediante prácticas deportivas y recreativas. La sinergia de estos procesos posteriores a los gobiernos neoliberales en Argentina y otras regiones de Latinoamérica se observan en las diversas apropiaciones del espacio público urbano y una suerte de retorno a las calles para vivir y disfrutar la ciudad, a pesar de los miedos, inseguridades, contaminaciones y zonas de caos discontinuo que se encarnan en agorafobias urbanas de todo tipo (Borja y Muxí, 2000).

De hecho, en la actualidad existen sobrados ejemplos de gobiernos municipales, provinciales y nacionales que intervienen el espacio urbano en, básicamente, dos líneas jerarquizadas que se manifiestan como tendencias, al menos, en Latinoamérica: a) infraestructura y recursos para la práctica de actividad física y deporte recreativo orientado a la salud, y b) infraestructura y recursos para prácticas y deportes emergentes -a veces llamados alternativos- realizados, preferencialmente, por niños y jóvenes. En los límites de este artículo, se tratará la primera tendencia, intentando dar forma al concepto de prácticas corporales desde los usos y sentidos que se le atribuyen en las políticas municipales y provinciales de recuperación del espacio público.

\section{MAPAS, PROCESOS Y DESPLAZAMIENTOS}

En la particularidad de la problemática propuesta, el énfasis que los gobiernos municipal y provincial realizan sobre la construcción de la idea de Estado presente se asienta en la crítica al corrimiento de las responsabilidades públicas por parte de los gobiernos neoliberales que los precedieron. En respuesta, estas políticas presentan al Estado y sus instituciones dependientes como instancia legítima y, por tanto, garante en el diseño, gestión, implementación y evaluación de las políticas de planificación y desarrollo urbano. En este sentido, las políticas estatales (Oszlak y O’Donell, 1995) no pueden ser desvinculadas de los gobiernos que las producen (Lahera, 2004) por lo que, tal como se aclaraba en el párrafo anterior, resulta necesario reconstruir las coyunturas particulares en las que se desarrollan.

Los PREP representan uno de los ejes principales de las políticas tanto municipales como provinciales desde el año 2007. Las primeras acciones políticas municipales se realizaron en un marco de debate intenso y controversias entre múltiples actores e instituciones -autoridades municipales y provinciales, instituciones civiles, grupos de profesionales e intervenciones de la Corte Bonaerense de Justicia- por el establecimiento de un nuevo Código de Ordenamiento Urbano (COU) en la ciudad de La Plata. Es decir, estas políticas impulsaron transformaciones de la ciudad en un momento en que sus marcos legales estaban siendo fuertemente cuestionados. Sin embargo, la atención y tensión estuvieron puestas en la construcción de viviendas y en los beneficios que el parche -así se denominaba comúnmente a la reforma- realizado por el gobierno municipal representaba a los grandes emprendimientos inmobiliarios de la zona. En ese primer momento, las discusiones no versaron en la transformación y los usos de los espacios verdes de la ciudad de La Plata.

En efecto, las intervenciones políticas de los espacios verdes del casco urbano de la ciudad de La Plata comienzan en el año 2007 y se acentúan en 2008, alcanzando su máxima expresión en los meses previos a las elecciones legislativas del año 2009. Estas observaciones denotan la centralidad de la cuestión espacio público en las políticas locales y, por otros indicios, en las provinciales de ese mismo periodo. El cuadro 1 sistematiza algunas de estas cuestiones, poniendo de relieve marcos institucionales, propósitos y la tríada sujetos/espacios/prácticas reconocidas en las políticas de recuperación del espacio público. 


\begin{tabular}{|c|c|c|}
\hline Prep & La Plata & Provincia de Buenos Aires \\
\hline Marco institucional & $\begin{array}{l}\text { 1. Dirección de Obras Públicas (2007-2009) } \\
\text { 2. Subsecretaría de Espacio Público e Higiene } \\
\text { (2009-2013) } \\
\text { 3. Secretaría de Espacio Público (2014) }\end{array}$ & $\begin{array}{l}\text { 1. Programa Soluciones iya! } \\
\text { Jefatura de gabinete en coordinación con la Fun- } \\
\text { dación Banco Provincia y ministerios provinciales } \\
\text { (2007-2011) } \\
\text { 2. Secretaría de Espacio Público (2012-2014) }\end{array}$ \\
\hline Objetivo general & $\begin{array}{l}\text { "Renovar completamente los parques y plazas } \\
\text { para que todos los vecinos puedan disfrutar de los } \\
\text { circuitos aeróbicos, los juegos integradores y los } \\
\text { sectores de descanso" (Entrevista, 08/11/2012). }\end{array}$ & $\begin{array}{l}\text { "Realizar un cambio trascendental en el espa- } \\
\text { cio, fundamentalmente en las plazas, en su uso y } \\
\text { estado, para propiciar las actividades recreati- } \\
\text { vas, culturales y deportivas" (Gacetilla de Prensa, } \\
30 / 10 / 2008 \text { ). }\end{array}$ \\
\hline Sujetos destinatarios & $\begin{array}{l}\text { "Niños; niños con capacidades diferentes; veci- } \\
\text { nos; familia; abuelos". }\end{array}$ & $\begin{array}{l}\text { "Jóvenes; niños; familia; vecinos; personas } \\
\text { discapacitadas". }\end{array}$ \\
\hline Espacios recuperados & $\begin{array}{l}\text { Parques; plazas; ramblas; barrios; espacios } \\
\text { verdes. }\end{array}$ & $\begin{array}{l}\text { Plazas; jardines; playones; plazoletas; áreas ver- } \\
\text { des; barrios. }\end{array}$ \\
\hline Prácticas promovidas & $\begin{array}{l}\text { Juegos infantiles; ejercicios de flexibilidad y mus- } \\
\text { culación; actividades "aeróbicas"; deportes. }\end{array}$ & Deportes; recreación; juegos infantiles. \\
\hline
\end{tabular}

Una lectura de los marcos institucionales en tanto procesos políticos evidencia un creciente desarrollo institucional de la cuestión espacio público en ambos niveles de gobierno. En el caso municipal, las políticas de espacio público comenzaron como responsabilidad de una Dirección y en el periodo estudiado se produjo su traspaso a Subsecretaría. En tanto, en el Gobierno de la provincia de Buenos Aires, la recuperación del espacio público comenzó como arista de un programa y, con la posterior creación de la Secretaría de Espacio Público en el año 2012, se institucionalizó como dependencia provincial específica.

El año 2012 sería el punto de inflexión en las acciones del gobierno municipal ya que, en el verano 2011-2012, se lanza la campaña "La Plata Ciudad Saludable"3. Esta campaña contó con especial participación de la Dirección de Deportes y Recreación por una política específica denominada "Programa Plazas Saludables". Según las propagandas, este programa está destinado a toda la población con el objetivo común que es el aprovechamiento del espacio público de forma saludable, deportiva y recreativa. Como dato relevante y que la diferencia de otras políticas similares implementadas en otras localidades del país, se destaca el hecho de que los participantes del programa cuentan con el asesoramiento

3 Según la información oficial difundida en gacetillas y en medios locales, esta es una campaña coordinada por la Jefatura de Gabinete de la Municipalidad de La Plata que tiene como objetivo brindar un lugar de esparcimiento, recreación y deporte en los espacios verdes del casco urbano de la ciudad. La Dirección de Deportes y Recreación, la Subsecretaría de Salud y Medicina Social desarrollan esta actividad en forma simultánea en seis espacios verdes de la ciudad. directo de profesores en educación física en las distintas estaciones saludables de determinadas plazas.

Además, la orientación de estas políticas es nutrida por otras vías de gestión como: a) la ordenanza municipal "Chau baldío"4; elemental en la recuperación de la plaza y el polideportivo del barrio Ripoll (Gran La Plata), que constituye una referencia ineludible y emblema de la gestión local, y b) el Presupuesto Participativo, en el que los mismos vecinos votan propuestas de obras mediante asambleas barriales. En este último ítem se destaca el caso del Parque Castelli ya que el proyecto ganador de una de las primeras convocatorias solicitó explícitamente la instalación de juegos infantiles, del circuito aeróbico y de los elementos que conforman el gimnasio al aire libre.

A modo de síntesis provisoria, con lo expuesto resulta claro que para ambos gobiernos -municipal y provincial- la recuperación del espacio público es una cuestión elemental en sus acciones políticas de reforma de la ciudad. En consecuencia, el cuadro presentado intenta graficar el creciente grado de institucionalización que ha cobrado esta cuestión. Fundamentalmente, interesa resaltar que los principales usos del espacio urbano

4 El artículo más representativo de la mencionada ordenanza es el número 13 ya que establece: “[...] la autoridad de aplicación, mediante convenio con los titulares de dominio, podrá afectar inmuebles ubicados en el radio del partido para destinarlos al emplazamiento de espacios verdes, parquización, plazas o centros comunitarios, cuyo mantenimiento estará a cargo del municipio durante el periodo de cesión del predio, el cual deberá computarse desde la toma de posesión por parte del municipio hasta el vencimiento del convenio. La cesión no podrá ser inferior a tres (3) años". 
promovidos por estos gobiernos están asociados a prácticas corporales que abarcan el amplio espectro entre lo deportivo, lo recreativo y la actividad física orientada a la salud.

\section{LAS PRÁCTICAS CORPORALES EN LA "RECUPERACIÓN DEL ESPACIO PÚBLICO" PLATENSE}

Ante la vaguedad del término prácticas corporales, utilizado en campos y estudios diversos para nombrar objetos diferentes, es necesario explicitar su especificidad en cuanto categorías analíticas. En este caso, las prácticas corporales se definen entre las discursividades de las políticas estudiadas y la materialidad de aquellas prácticas observadas en el espacio urbano, por tanto, no se elaboran completamente a priori. Sin embargo, la potencialidad inicial que esta categoría analítica algo más plástica que la de deporte o práctica deportiva reside en que permite trascender la distinción por fines entre deporte y recreación que divide al campo deportivo (Levoratti, 2013a) y así incluir una variedad de prácticas emergentes que escapan a esas definiciones y que, sin embargo, las interpelan. En resumidas palabras, con Gabriel Cachorro et al. (2010) entendemos a las prácticas corporales como expresiones del movimiento modeladas por culturas de lo corporal, por ende, abiertas a las contingencias subjetivas e históricas de las cuales son producto y productoras.

Para introducir el análisis de los usos y sentidos de las prácticas corporales en estas políticas se presentan algunos fragmentos de entrevista con el citado "José". En principio, estos breves fragmentos de entrevista dan cuenta de las construcciones de sentido en relación al espacio público y a los posibles usos corporales. Como contexto de estas cuestiones, este funcionario municipal afirma que las PREP se asientan sobre un diagnóstico político de abandono, atraso, desuso, peligrosidad:

Lamentablemente en anteriores gestiones, que se ha hecho mucho también en obras públicas, el espacio público no se ha tenido en cuenta como algo que es parte de la cultura del platense, porque la ciudad nació, se concibió con esta idea de una ciudad que contemple el esparcimiento, la recreación, el verde; bueno, eso no se tuvo en cuenta durante mucho tiempo, con lo cual el uso, el abuso, hizo que mucho de los espacios públicos terminaran en condiciones casi inhabitables, la gente prácticamente no iba. [...] No había una política de espacio público concretamente. (Entrevista realizada el 8 de noviembre de 2012).

Diez años atrás, se habían convertido en tierra de nadie, uno veía una plaza, y no podía ir, porque había una banda de pibes que a la gente le daba miedo. (Entrevista realizada el 3 de diciembre de 2013).

Por otro lado, la propaganda que a continuación ilustra estas líneas también retrata algunos elementos de estas operaciones materiales y simbólicas sobre la ciudad. Entre estas se destacan el acondicionamiento de sectores verdes, la instalación de luminarias y de juegos que, en este caso, es reforzada con la imagen de dos niñas en una de las obras construidas como condición primera de visibilidad. Pero estas obras destinadas al juego infantil no fueron las únicas realizadas. Una fotografía de las cartelerías que se instalaban en cada espacio recuperado, tomada en el año 2009 en las inmediaciones del Parque San Martín, permite reconstruir el hecho de que también se construyeron "circuitos aeróbicos con módulos de elongación y musculación, juegos para niños integradores, áreas deportivas integradoras, y plazas de encuentro", entre otras obras.

Ilustración 1. Propaganda del PREP en medios gráficos (2010).

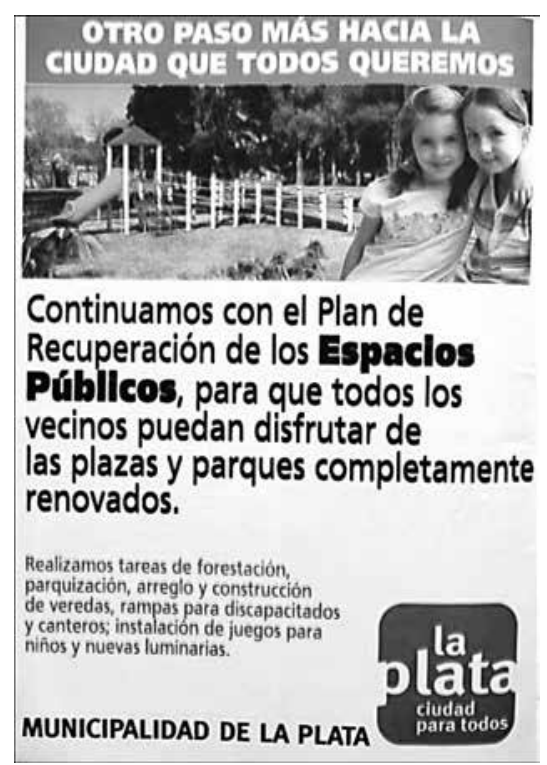

Por esta razón, es posible afirmar que el PREP municipal promueve un amplio espectro de usos del espacio urbano y de los espacios verdes de la ciudad, entre ellos: a) juegos infantiles en estructuras dispuestas con fines integradores; b) actividades orientadas a obtener beneficios en la zona metabólica aeróbica; c) ejercicios en estructuras de hierro -llamadas módulos- que permiten estimular las capacidades flexibilidad y fuerza; d) deportes en áreas creadas con fines similares a los enunciados en los juegos integradores. 
Ilustración 2. Fotografías del circuito aeróbico y módulos de la rambla de la avenida-circunvalación 72 (2012).

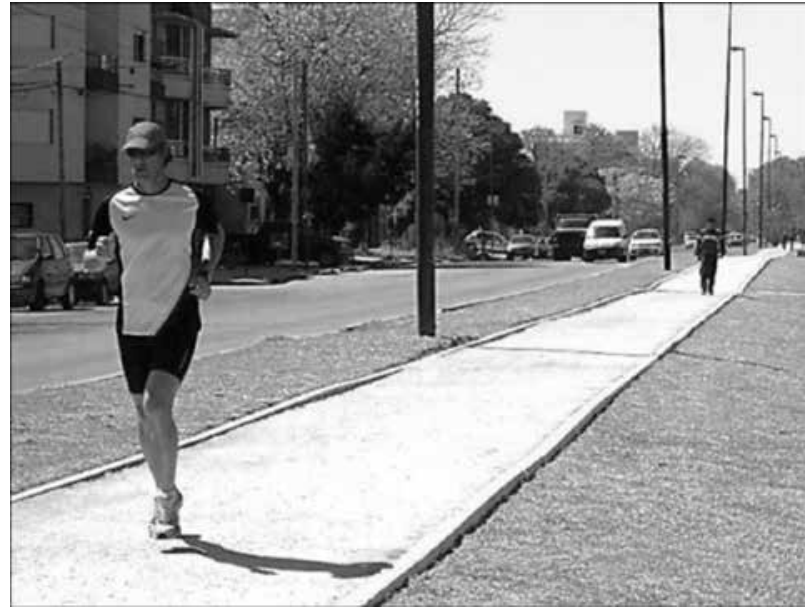

El uso político que se hace del término deporte, es decir, en tanto categoría social construida en el seno de discursos y acciones gubernamentales, constituye una de las diferencias primordiales entre los dos PREP. Mientras que el PREP provincial hace hincapié en la práctica deportiva orientada al rendimiento o competencia casi de forma exclusiva, el municipal privilegia en tal concepción de deporte la orientación recreativa e incluye sistemáticamente en esa misma definición a la práctica de actividad física con fines saludables. En estas condiciones de posibilidad, también emergen los juegos infantiles y los sectores para niños con necesidades especiales como sujetos legítimos de carácter municipal. En cambio, en el ámbito provincial son los jóvenes los sujetos mencionados como destinatarios privilegiados de las prácticas deportivas.

El espectro de prácticas corporales -entre las que se encuentran las deportivas-, promovido por el Gobierno municipal a partir de la reforma del espacio urbano, constituye un refuerzo de los sentidos expuestos en los fragmentos de entrevista a comienzos del apartado, ya que se presenta como la solución política al diagnóstico de abandono de la ciudad y de desuso del espacio urbano. En efecto, la traducción de sentidos de las prácticas corporales en la ciudad (Cachorro, 2009) en relación a las características gubernamentales que las promueven dotan de especificidad a estas construcciones de sentido en el marco de una cultura política de lo corporal altamente atravesada por las tradiciones fundacionales características de la ciudad de La Plata: el racionalismo y el higienismo que modelaron inicialmente sus formas; que dotaron en sus orígenes

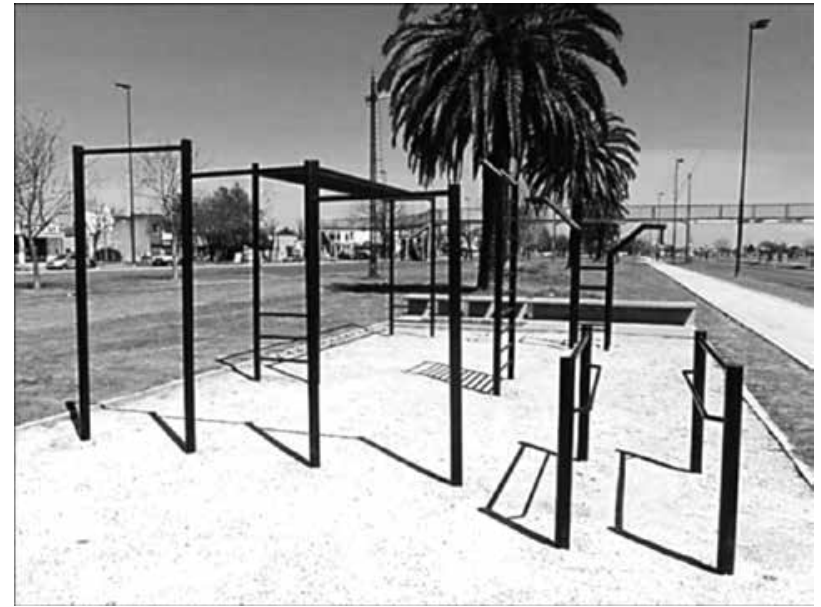

decimonónicos al cuadrado del casco urbano de un espacio verde cada seis cuadras. ${ }^{5}$

Esta observación conecta con el hecho de que se liga de inmediato la cuestión del espacio público a una cultura platense modelada por las tradiciones fundacionales de la ciudad. Esta relación es una constante en materiales empíricos diversos por lo que no puede dejar de ser pensada como una estrategia discursiva dominante que apela a las tradiciones y las incorpora como elementos residuales (Williams, 2012) con el propósito de construir legitimidad política. En esta búsqueda de legitimidad, la definición y promoción de prácticas corporales cumplen un rol fundamental en la medida que intenta conectar la dimensión proyectiva/planificada de la ciudad con la dimensión fenomenológica, efectiva, vivenciada o experienciada de la ciudad que solo es posible a través/por/en el cuerpo.

\section{USOS, SENTIDOS Y TIPOLOGÍAS}

En términos generales, los usos y sentidos de las prácticas corporales en el espacio urbano constituidos desde ambos sectores de la política -provincial y municipalrevisten y aglutinan esta diversidad de prácticas bajo el término deporte. En el ámbito municipal, los sentidos gubernamentales de deporte refieren a la práctica recreativa y de actividad física disolviendo su componente competitivo y colaborando con el mítico binomio deporte es igual a salud. Sin embargo, la recreación o lo recreativo emerge como un conjunto de prácticas

5 Para ahondar estos aspectos urbanísticos se recomienda consultar a Segura (2009). 
raramente especificadas en ambos sectores de la política. Por lo general es representado vagamente como actividad del tiempo libre, no productiva, asociada al juego pero no al jugar como posibilidad subjetiva o grupal de re-crearse, es decir, como práctica del ocio. En línea con estas regularidades y observaciones, se presentarán de modo breve y esquemático algunas sistematizaciones que resultaron de estas primeras indagaciones. Cabe mencionar que las tipologías no son construcciones analíticas cerradas y se exponen por la crítica que habilitan a posteriori.

\section{El deporte en el espacio público como derecho}

Esta concepción encuentra su expresión más común y visible en el deporte para todos, asociado a la noción liberal de espacio público como espacio de prácticas libres e irrestrictas en la ciudad. Sin embargo, esta es una versión legalista y abstracta tanto del deporte como de la ciudad, en la medida que borra o invisibiliza los conflictos en el espacio urbano y las diferencias que tanto a priori como en efecto existen en sus posibilidades de apropiación. En consecuencia, lo que está en juego en este uso y sentido es la noción de ciudadanía mediada por el deporte. De hecho, la ordenanza municipal 9880 -Código de Ordenamiento y Uso del Espacio Público- refuerza desde esta perspectiva los usos legítimos e ilegítimos del espacio urbano que el espectro gubernamental de prácticas corporales propone.

El deporte y la recreación en el espacio público como forma de encuentro y reconocimiento vecinal/familiar

Este uso político del deporte y la recreación se desprende de la primera tipología pero la especifica en cuanto detalla los fines positivos de las relaciones políticamente deseadas. Esta cuestión puede ser interpretada desde Elías y Dunning (1992) como característica propia del desarrollo de los Estados modernos en el proceso civilizatorio de Occidente. En este proceso, la relevancia social del fenómeno deportivo y de las prácticas deportivas-recreativas reside en su potencia identificatoria y de sociabilidad, descentrando a la religión y al trabajo como núcleos de estas estas funciones sociales y culturales.

La particularidad del caso La Plata reside en que estas formas de sociabilidad e identificación están mediadas por las características urbanísticas de la ciudad, que permiten tanto el desarrollo de diversas prácticas corporales al aire libre como de modos particulares de construir comunidad(es). Estas características urbanas no definen dichos modos, pero sí consisten en condiciones de posibilidad extraordinarias. En este contexto, la operación discursiva sobre la familia y lo vecinal intenta desplazar a la arena de lo público en la ciudad al núcleo de socialización que, tal como afirma Richard Sennett (1978), es históricamente identificado con lo privado y la intimidad. Estas invocaciones a los núcleos básicos de la ciudadanía son, tal vez, intentos por reconstruir o reponer desde otra arista las fracturas institucionales de la modernidad en el mundo contemporáneo.

\section{El deporte en el espacio público como "escuela de vida"}

Este uso y sentido está más vigente en las discursividades del nivel provincial, pero se encuentra de modo subyacente en el PREP municipal. Las relaciones entre deporte y valores anclados en las moralidades de ciertas políticas socioeducativas de la provincia de Buenos Aires han sido investigadas y descritas en detalle por Alejo Levoratti (2013b). En la problemática del espacio público urbano estas moralidades aparecen como extensión del deporte institucionalizado e intentan trasladarse a los espacios extramuros. Estas operatorias se observan en la proliferación de clínicas o charlas realizadas en espacios recuperados -a veces en las mismas inauguraciones- que son protagonizadas por deportistas reconocidos en el ámbito nacional, cuya intervención en el espacio público se fundamenta en la transmisión de una experiencia educativa/formativa. En efecto, no se considera casualidad sino estrategia el hecho de que ambas políticas insistan sobre sujetos en edades escolarizadas.

\section{El deporte y la actividad física como profilaxis}

Este sentido se asienta sobre la ecuación movimiento es igual a salud, mediada por el paradigma preventivo en salud pública. La relación mencionada se traduce en la búsqueda de un "estilo de vida activo" (Fraga, 2008), donde movimiento y gasto de calorías equivalen a salud, mientras que la morbilidad de los cuerpos estáticos y sedentarios representa su temido opuesto: la enfermedad. En esta operatoria de demonización del sedentarismo y glorificación de la vida activa convergen tanto los medios masivos de comunicación como las industrias culturales (De Carvalho, 1998), sobre todo en la producción y circulación de imágenes que 
exhiben tipos ideales de cuerpo y de comportamientos saludables. Respecto del lenguaje, Mainetti $(2006$ advierte sobre la medicalización en la vida cotidiana y la penetración del ideal de bienestar con la conformación de una cultura de la salud, en la que se podrían inscribir estas prácticas corporales profilácticas.

\section{El juego como herramienta de integración social}

Este uso y sentido de las prácticas corporales lúdicas hace referencia explícita a un debate central de lo contemporáneo, que es el reconocimiento y tratamiento de la diversidad. Sin embargo, la política lo reduce a las diferencias entre niños con capacidades diferentes o personas discapacitadas basadas en algún ideal implícito de normalidad construido en la plena disponibilidad motriz o ausencia de dificultades motrices para jugar. Por ello es que el énfasis está puesto en el espacio, en la estructura del juego y no en el jugar como modo subjetivo y relacional del cuerpo en el espacio. Desde esta perspectiva, lo que integra a los sujetos es el espacio per se y no las prácticas o los lazos sociales que a través de las prácticas se efectivizan en esos espacios. La materialidad de la disposición espacial de estos juegos evidencia una tendencia a clasificar y a sectorizar a los niños según estas características aunque discursivamente se procure lo contrario.

Para finalizar, y en tanto estas tipologías no constituyen constructos cerrados, se aclara que la segunda parte de esta investigación se encuentra en desarrollo y hace hincapié en las apropiaciones concretas del espacio urbano por parte de sujetos y grupos deportivosrecreativos en la ciudad, destacando las interpelaciones a las políticas analizadas en estas líneas. En efecto, se argumenta: "[...] lo que da su verdadera forma a una ciudad no son las arquitecturas ni las ingenierías sino los ciudadanos" (Martín Barbero, 2008, p. 217).

\section{BREVES CONSIDERACIONES FINALES}

Lo que se observa grosso modo en los señalados usos y sentidos gubernamentales es que el espectro de prácticas corporales tiende a fundirse en un concepto lato de deporte, que lo vacía de su sentido más característico: el del agon o competencia. Estas políticas, en donde los usos y sentidos indican que casi todo es deporte, se inscriben en la legitimidad que proveen los registros de significación sedimentados en la vida cotidiana o más conocidos como registros de sentido común. Algo similar sucede con las relaciones entre espacio público y espacio urbano. A menudo, estas suelen confundirse y utilizarse como sinónimos por el hecho de que, en su concepción clásica, son términos emparentados por su genealogía occidental. Como señala Jean-Marc Ferry (2001) la historia del concepto espacio público se remonta, en su concepción antigua, al agora -la plazay la polis -la ciudad- ateniense.

Las prácticas corporales asociadas a esta concepción del espacio público aparecen en el corpus de estas políticas como actividades y espacios de uso libre y gratuito. Como se ha mencionado en el apartado anterior, esta caracterización de las prácticas en el espacio público como derecho ciudadano enmascara tanto la dimensión conflictiva de las relaciones entre ciudad y corporalidades como el carácter procesual de lo público. Por el contrario, como afirma Gabriel Cachorro (2009), las prácticas corporales realizadas por sujetos y grupos en la ciudad también construyen ciudadanía. Es decir, desde una perspectiva sociocultural del espacio público urbano, los marcos legales y políticos solo constituyen las garantías abstractas o las condiciones de posibilidad sobre las que se practica la ciudadanía, sea acatando las producciones políticas del espacio urbano en cuanto bien público o interpelando estas propuestas a través de otras prácticas corporales. 


\section{REFERENCIAS BIBLIOGRÁFICAS}

Borja, J. y Muxí, Z. (2000). El espacio público: ciudad y ciudadanía. Barcelona: Electa.

Buenos Aires (2012). Decreto 112/12. Creación de la Secretaría de Espacio Público de la provincia de Buenos Aires.

Cachorro, G. (2009). Prácticas corporales. Traducción de sentidos en la ciudad. Pensar a Prática 12(2), 1-10.

Cachorro, G. Césaro, A.R., Scarnatto, M., Villagrán, J.P. (2010). La ciudad, los jóvenes y el campo de las prácticas corporales. Revista Brasileira do Ciências do Esporte 31(3), 43-58.

De Carvalho, Y. M. (1998). El "mito" de la actividad física y la salud. Buenos Aires: Lugar Editorial.

Ferry, J. M. (2001). Las transformaciones de la publicidad política. En Ferry J.M. y Wolton, D. (comp.). El nuevo espacio de lo público. Barcelona: Gedisa.

Fraga, B. A. (2008). Estilo de vida activo: un nuevo orden físico-sanitario. En: P. Scharagrodsky (comp.). Gobernar es ejercitar. Fragmentos históricos de la Educación Física en Iberoamérica (pp.169-176). Buenos Aires: Editorial Prometeo.

Gorelik, A. (2008). El romance del espacio público. Alteridades 18(36), 33-45.

Guber, R. (2001). La etnografía. Método, campo y reflexividad. Bogotá: Norma.

Honorable Concejo Deliberante de La Plata. (2008). Ordenanza 10.459: "Chau baldío".

Honorable Concejo Deliberante de La Plata. (2005). Ordenanza 9880: Código de Ordenamiento y Uso del Espacio Público.

Lahera, E. (2004). Política y políticas públicas. En: CEPALSerie Políticas sociales, 95. Santiago de Chile.
Levoratti, A. (2013a). Deporte recreativo o social. La competencia dividiendo al campo deportivo. En: J. Branz, J. Garriga Zucal y V. Moreira (comp.). Deporte y Ciencias Sociales. Claves para pensar las sociedades contemporáneas (pp. 265-292). La Plata: EDULP.

Levoratti, A. (2013b). Disputas por el sentido del deporte en programas de política socioeducativa. Un análisis sobre sus representaciones y prácticas en funcionarios estatales y profesores de educación física (Provincia de Buenos Aires, 2004-2011). Tesis inédita de Maestría. Buenos Aires: Universidad Nacional de San Martín.

Mainetti, J. A. (2006). La medicalización de la vida y el lenguaje. Electroneurobiológica 14(3), 71-89.

Marradi, A.; Archenti, N. y Piovani, J. (2007). Metodología de las Ciencias Sociales. Buenos Aires: Emecé Editores.

Martín Barbero, J. (2008). Lo público. Experiencia urbana y metáfora ciudadana. Cuadernos de Información y Comunicación, 13, 213-226.

Oszlak, O. y O’Donell, G. (1995). Estado y políticas estatales en América Latina: hacia una estrategia de investigación. Redes 2(4), 99-128.

Reguillo, R. (2012). Los estudios culturales. El mapa incómodo de un relato inconcluso. Revista de Estudios para el Desarrollo Social de la Comunicación 1(2), 189199.

Segura, R. (2009). La persistencia de la forma (y sus omisiones). Un estudio del espacio urbano de la ciudad de La Plata a través de sus ciudades análogas. Cuadernos de Antropología Social, 30, 173-197.

Taylor, S. y Bogdan, R. (1987). Introducción a los métodos cualitativos de investigación. La búsqueda de significados. Barcelona: Paidós.

Williams, R. (2012). Cultura y Materialismo. Buenos Aires: La Marca Editora. 